\title{
An Extension of the Exponential Formula in Enumerative Combinatorics
}

\author{
Gilbert Labelle and Pierre Leroux \\ LACIM, Dép. de mathématiques, Université du Québec à Montréal \\ C.P. 8888, Succ. Centre-Ville, Montréal (Québec), Canada H3C 3P8
}

Submitted: March 11, 1995; Accepted: July 15, 1995

En hommage à Dominique Foata, à l'occasion de son soixantième anniversaire.

\begin{abstract}
Let $\alpha$ be a formal variable and $F_{w}$ be a weighted species of structures (class of structures closed under weight-preserving isomorphisms) of the form $F_{w}=E\left(F_{w}^{c}\right)$, where $E$ and $F_{w}^{c}$ respectively denote the species of sets and of connected $F_{w}$-structures. Multiplying by $\alpha$ the weight of each $F_{w^{-}}^{c}$ structure yields the species $F_{w(\alpha)}=E\left(F_{\alpha w}^{c}\right)$. We introduce a "universal" virtual weighted species, $\Lambda^{(\alpha)}$, such that $F_{w^{(\alpha)}}=\Lambda^{(\alpha)} \circ F_{w}^{+}$, where $F_{w}^{+}$denotes the species of non-empty $F_{w^{-}}$-structures. Using general properties of $\Lambda^{(\alpha)}$, we compute the various enumerative power series $G(x), \widetilde{G}(x)$, $\bar{G}(x), G(x ; q), G\langle x ; q\rangle, Z_{G}\left(x_{1}, x_{2}, x_{3}, \ldots\right), \Gamma_{G}\left(x_{1}, x_{2}, x_{3}, \ldots\right)$, for $G=F_{w^{(\alpha)}}$, in terms of $F_{w}$. Special instances of our formulas include the exponential formula, $F_{w^{(\alpha)}}(x)=\exp \left(\alpha F_{w}(x)\right)=\left(F_{w}(x)\right)^{\alpha}$, cyclotomic identities, and their $q$-analogues. The virtual weighted species, $\Lambda^{(\alpha)}$, is, in fact, a new combinatorial lifting of the function $(1+x)^{\alpha}$.

\section{Résumé}

Soit $\alpha$ une variable formelle et $F_{w}$ une espèce de structures pondérée (classe de structures fermée sous les isomorphismes préservant les poids) de la forme $F_{w}=E\left(F_{w}^{c}\right)$, où $E$ et $F_{w}^{c}$ désignent respectivement l'espèce des ensembles et celle des $F_{w}$-structures connexes. En multipliant par $\alpha$ le poids de chaque $F_{w}^{c}$-structure, on obtient l'espèce $F_{w^{(\alpha)}}=E\left(F_{\alpha w}^{c}\right)$. Nous introduisons une espèce virtuelle "universelle", $\Lambda^{(\alpha)}$, telle que $F_{w^{(\alpha)}}=\Lambda^{(\alpha)} \circ F_{w}^{+}$, où $F_{w}^{+}$désigne l'espèce des $F_{w}$-structures non-vides. En faisant appel à des propriétés générales de $\Lambda^{(\alpha)}$, nous calculons les diverses séries formelles énumératives $G(x), \widetilde{G}(x), \bar{G}(x), G(x ; q), G\langle x ; q\rangle, Z_{G}\left(x_{1}, x_{2}, x_{3}, \ldots\right), \Gamma_{G}\left(x_{1}, x_{2}, x_{3}, \ldots\right)$, de $G=F_{w^{(\alpha)}}$, en fonction de $F_{w}$. Comme cas spéciaux des formules que nous développons, on retrouve la formule exponentielle, $F_{w^{(\alpha)}}(x)=\exp \left(\alpha F_{w}(x)\right)=\left(F_{w}(x)\right)^{\alpha}$, les identités cyclotomiques, ainsi que leurs $q$-analogues. L'espèce virtuelle pondérée, $\Lambda^{(\alpha)}$, est, en fait, un nouveau relèvement combinatoire de la fonction $(1+x)^{\alpha}$.
\end{abstract}

\section{Introduction}

Let $f(x)$ and $g(x)$ be two functions or formal power series such that

$$
f(x)=\exp (g(x))
$$

The expression $f(x)^{\alpha}$ can then be given a meaning, for any variable $\alpha$, by means of the "exponential formula"

$$
f(x)^{\alpha}=\exp (\alpha g(x))
$$




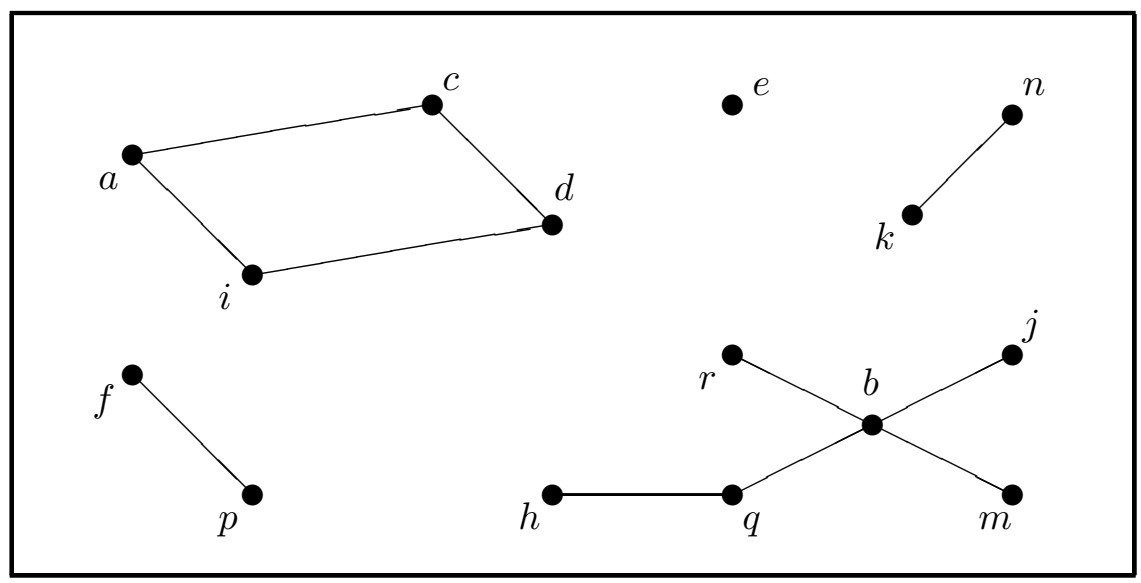

Figure 1: A graph $g$ with $w(g)=y^{11}$ and $w^{(\alpha)}(g)=\alpha^{5} y^{11}$

D. Foata played a pioneer role in the interpretation and use of these formulas in enumerative combinatorics; see for example [3], [12], [16]. Essentially, if $f(x)$ and $g(x)$ are the exponential generating series for the structure classes $F$ and $G$, then equation (1) expresses the fact that the $F$-structures can be identified with assemblies of $G$-structures, the latter being considered as "connected" $F$-structures. In that case, the "weight" $\alpha^{c(s)}$ can be assigned to any $F$-structure $s$, where $c(s)$ denotes the number of "connected components" of $s$; one says that the variable $\alpha$ acts as a counter for (or marks) the connected components. Formula (2) then gives the exponential generating series of this new weighted class. For example, it is immediately seen that the function $(1-x)^{-\alpha}$ is the generating series for permutations, where $\alpha$ acts as a cycle counter. This approach is fundamental in the development of combinatorial models for the classical orthogonal polynomials; see, for example, [13]-[15], [17].

In the following, we consider weighted structure classes (called species, see [2], [19], [24]) that are closed under isomorphisms induced by relabellings, that is, by bijections of the underlying sets. In that case, new generating series, symmetric functions (cycle index series), and $q$-analogues can be associated, in relation with the enumeration of unlabelled (i.e., up to isomorphism) or asymmetric (i.e., whose automorphism group is reduced to the identity) structures. When moreover the concept of connected components can be introduced, there arises the question of determining the relations analogous to (2) for these various series. As we will see, more complex exponential formulas appear, involving polynomials in $\alpha$ related to the classical "cyclotomic identity" and to a new "cocyclotomic identity" (see Corollary 2.3).

Let us illustrate our approach with the species $\mathrm{Gr}_{w}$ of (simple) graphs, weighted by an edge counter, $y$. For instance, the graph $g$ of Figure 1 has weight $w(g)=y^{11}$ since it has 11 edges. Note that this weighting is invariant under relabellings and multiplicative with respect to the natural concept of connected components, in the sense that the weight of a graph is equal to the product of the weights of its components. These facts are summarized by the equation $\mathrm{Gr}_{w}=E\left(\mathrm{Gr}_{w}^{c}\right)$, where $E$ denotes the species of sets and $\mathrm{Gr}^{c}$ denotes the connected graphs. Now the weight $w$ can be refined to a new weight $w^{(\alpha)}$ by adjoining a counter $\alpha$ of connected components. Hence the previous graph $g$ has a refined weight $w^{(\alpha)}(g)=\alpha^{5} y^{11}$ (see Figure 1). This refinement $w \mapsto w^{(\alpha)}$ transforms the species $\mathrm{Gr}_{w}$ of graphs weighted by the number of edges into the species $\mathrm{Gr}_{w}(\alpha)$ of graphs weighted by the numbers of edges and of connected components. Since any graph is an assembly of connected graphs, 
the transformation can be described as

$$
\mathrm{Gr}_{w}=E\left(\mathrm{Gr}_{w}^{c}\right) \mapsto \mathrm{Gr}_{w^{(\alpha)}}=E\left(\mathrm{Gr}_{\alpha w}^{c}\right),
$$

where $\alpha w$ expresses that the weight $w$ of any connected component is multiplied by $\alpha$.

It is clear that the transformation (3) can be extended to any weighted species $F_{w}$, of the form $F_{w}=E\left(F_{w}^{c}\right)$, where $F_{w}^{c}$ denotes the species of connected $F_{w}$-structures:

$$
F_{w}=E\left(F_{w}^{c}\right) \mapsto F_{w^{(\alpha)}}=E\left(F_{\alpha w}^{c}\right),
$$

Our goal is to analyse the general transformation $F_{w} \mapsto F_{w^{(\alpha)}}$ and to deduce a number of enumerative results. We show, in section 2, that there exists a "universal" virtual (i.e., formal difference of species, see [2], [20], [36]) weighted species, $\Lambda^{(\alpha)}$, such that

$$
F_{w^{(\alpha)}}=\Lambda^{(\alpha)} \circ F_{w}^{+}
$$

where $F_{w}^{+}=F_{w}-1$ denotes the species of non-empty $F_{w}$-structures, and we compute the main enumerative series associated with $\Lambda^{(\alpha)}$. From this follows an explicit general form for the series $G(x), \widetilde{G}(x), \bar{G}(x), G(x ; q), G\langle x ; q\rangle, Z_{G}\left(x_{1}, x_{2}, x_{3}, \ldots\right), \Gamma_{G}\left(x_{1}, x_{2}, x_{3}, \ldots\right)$, associated with the species $G=F_{w^{(\alpha)}}$, in terms of the series associated with the species $F_{w}$. In section 3, we give a number of identities (and $q$-identities) which follow for some particular choices of species $F_{w}$. We conclude, in section 4 , with an analysis of the species $\Lambda^{(\alpha)}$. In particular, we give the first terms of the molecular decomposition of $\Lambda^{(\alpha)}$ which constitutes a new combinatorial lifting of the binomial formula for a formal exponent $\alpha$ :

$$
(1+x)^{\alpha}=1+\alpha x+\alpha(\alpha-1) \frac{x^{2}}{2 !}+\alpha(\alpha-1)(\alpha-2) \frac{x^{3}}{3 !}+\alpha(\alpha-1)(\alpha-2)(\alpha-3) \frac{x^{4}}{4 !}+\cdots .
$$

\section{The transformation $F_{w} \mapsto F_{w^{(\alpha)}}$}

Many formal power series can be assigned to a weighted species $F=F_{w}$. The two main ones, $Z_{F}$ and $\Gamma_{F}$ are called respectively the cycle index series of $F$ (see [19]) and the asymmetry index series of $F$ (see [23]-[25]). They are power series in an infinity of variables $x_{1}, x_{2}, x_{3}, \ldots$ :

$$
\begin{aligned}
& Z_{F}\left(x_{1}, x_{2}, x_{3}, \ldots\right)=\sum_{n=0}^{\infty} \frac{1}{n !} \sum_{\sigma \in S_{n}} f_{\sigma} x_{1}^{\sigma_{1}} x_{2}^{\sigma_{2}} x_{3}^{\sigma_{3}} \cdots, \\
& \Gamma_{F}\left(x_{1}, x_{2}, x_{3}, \ldots\right)=\sum_{n=0}^{\infty} \frac{1}{n !} \sum_{\sigma \in S_{n}} f_{\sigma}^{*} x_{1}^{\sigma_{1}} x_{2}^{\sigma_{2}} x_{3}^{\sigma_{3}} \cdots .
\end{aligned}
$$

In these formulas, the coeficients $f_{\sigma}$ (resp. $f_{\sigma}^{*}$ ) are $\mathbb{N}$-linear (resp. $\mathbb{Z}$-linear) combinations of the weights given to the $F$-structures on $[n]=\{1,2, \ldots, n\}, S_{n}$ is the symmetric group of degree $n$ and $\sigma_{k}$ denotes the number of cycles of length $k$ in the cyclic decomposition of any permutation $\sigma \in S_{n}$. In fact, $Z_{F}$ and $\Gamma_{F}$ can be seen as symmetric functions of auxiliary variables $\xi_{1}, \xi_{2}, \xi_{3}, \ldots$, by making the substitutions

$$
x_{i}:=p_{i}\left(\xi_{1}, \xi_{2}, \xi_{3}, \ldots\right)=\xi_{1}^{i}+\xi_{2}^{i}+\xi_{3}^{i}+\cdots, i \geq 1 \text { (power sums). }
$$

Other specializations of the variables $x_{1}, x_{2}, x_{3}, \ldots$, in the series (7)-(8) give (see [19], [23])

$$
Z_{F}(x, 0,0, \ldots)=\Gamma_{F}(x, 0,0, \ldots)=F(x)=\sum_{n \geq 0} f_{n} \frac{x^{n}}{n !},
$$




$$
\begin{aligned}
& Z_{F}\left(x, x^{2}, x^{3}, \ldots\right)=\widetilde{F}(x)=\sum_{n \geq 0} \widetilde{f}_{n} x^{n}, \\
& \Gamma_{F}\left(x, x^{2}, x^{3}, \ldots\right)=\bar{F}(x)=\sum_{n \geq 0} \bar{f}_{n} x^{n},
\end{aligned}
$$

where

$$
\begin{aligned}
f_{n} & =\text { total weight of the } F_{w} \text {-structures on }[n]=\{1,2, \ldots, n\} \\
\widetilde{f}_{n} & =\text { total weight of the unlabelled } F_{w} \text {-structures on } n \text { points, } \\
\bar{f}_{n} & =\text { total weight of the unlabelled asymmetric } F_{w} \text {-structures on } n \text { points. }
\end{aligned}
$$

Of course, when the weighting $w$ is trivial (i.e., $w=1$ for any structure), then the total weights (13) - (15) give the numbers of corresponding structures.

Finally, two $q$-series $F(x ; q)$ and $F\langle x ; q\rangle$ can be canonically associated to the species $F$ (see [7]-[9]) by setting

$$
\begin{aligned}
& F(x ; q)=\sum_{n=0}^{\infty} f_{n}(q) \frac{x^{n}}{n !_{q}}=Z_{F}\left(\frac{(1-q)}{(1-q)} x, \frac{(1-q)^{2}}{\left(1-q^{2}\right)} x^{2}, \frac{(1-q)^{3}}{\left(1-q^{3}\right)} x^{3}, \ldots\right) \\
& F\langle x ; q\rangle=\sum_{n=0}^{\infty} f_{n}\langle q\rangle \frac{x^{n}}{n !_{q}}=\Gamma_{F}\left(\frac{(1-q)}{(1-q)} x, \frac{(1-q)^{2}}{\left(1-q^{2}\right)} x^{2}, \frac{(1-q)^{3}}{\left(1-q^{3}\right)} x^{3}, \ldots\right)
\end{aligned}
$$

where

$$
n !_{q}=\frac{(1-q)}{(1-q)} \cdot \frac{\left(1-q^{2}\right)}{(1-q)} \cdot \frac{\left(1-q^{3}\right)}{(1-q)} \cdots \frac{\left(1-q^{n}\right)}{(1-q)}
$$

denotes the $q$-analogue of $n !\left(n !=\lim _{q \rightarrow 1} n !_{q}\right)$. These $q$-series satisfy the following properties:

$$
\begin{array}{ll}
\lim _{q \rightarrow 1} F(x ; q)=F(x), & \lim _{q \rightarrow 0} F(x ; q)=\widetilde{F}(x) . \\
\lim _{q \rightarrow 1} F\langle x ; q\rangle=F(x), & \lim _{q \rightarrow 0} F\langle x ; q\rangle=\bar{F}(x) .
\end{array}
$$

Moreover their coefficients, $f_{n}(q)$ and $f_{n}\langle q\rangle$, are polynomials in $q$ of degree $\leq n(n-1) / 2$. For $F=E$, the species of sets, the $q$-series (16) and (17) take the form of the two classical $q$-analogues of the exponential function:

$$
\begin{aligned}
& E(x ; q)=\sum_{n=0}^{\infty} \frac{x^{n}}{n !_{q}}=\frac{1}{(1-(1-q) x)(1-(1-q) q x)\left(1-(1-q) q^{2} x\right) \cdots} \\
& E\langle x ; q\rangle=\sum_{n=0}^{\infty} q^{n(n-1) / 2} \frac{x^{n}}{n !_{q}}=(1+(1-q) x)(1+(1-q) q x)\left(1+(1-q) q^{2} x\right) \cdots
\end{aligned}
$$

The definition of the series $Z_{F}, \Gamma_{F}, F(x), \widetilde{F}(x), \bar{F}(x), F(x ; q), F\langle x ; q\rangle$, can be extended to any virtual weighted species $F=F_{w}=A_{u}-B_{v}$, where $A_{u}$ and $B_{v}$ are two weighted species, by setting $Z_{F}=Z_{A_{u}}-Z_{B_{v}}, \Gamma_{F}=\Gamma_{A_{u}}-\Gamma_{B_{v}}$, and so on.

The transformations $F \mapsto Z_{F}$ and $F \mapsto \Gamma_{F}$, from species to index series, have the remarkable property of preserving the main combinatorial operations ([19], [23]): for any (virtual weighted) species $F$ and $G$, one has

$$
Z_{F+G}=Z_{F}+Z_{G}, \quad \Gamma_{F+G}=\Gamma_{F}+\Gamma_{G},
$$




$$
\begin{aligned}
Z_{F \cdot G}=Z_{F} \cdot Z_{G}, & \Gamma_{F \cdot G}=\Gamma_{F} \cdot \Gamma_{G}, \\
Z_{F \circ G}=Z_{F} \circ Z_{G}, & \Gamma_{F \circ G}=\Gamma_{F} \circ \Gamma_{G}, \\
Z_{F^{\prime}}=\frac{\partial Z_{F}}{\partial x_{1}}, & \Gamma_{F^{\prime}}=\frac{\partial \Gamma_{F}}{\partial x_{1}},
\end{aligned}
$$

In the right hand sides of (25), the operation used is the plethystic substitution of index series. It is defined, for two arbitrary index series $f_{w}\left(x_{1}, x_{2}, x_{3}, \ldots\right)$ and $g_{v}\left(x_{1}, x_{2}, x_{3}, \ldots\right)$, with $g_{v}(0,0,0, \ldots)=0$, arising from weighted virtual species $F_{w}$ and $G_{v}$, by

$$
\left(f_{w} \circ g_{v}\right)\left(x_{1}, x_{2}, x_{3}, \ldots\right)=f_{w}\left(\left(g_{v}\right)_{1},\left(g_{v}\right)_{2},\left(g_{v}\right)_{3}, \ldots\right)
$$

where $\left(g_{v}\right)_{k}:=g_{v^{k}}\left(x_{k}, x_{2 k}, x_{3 k}, \ldots\right)$ denotes the index series obtained by raising to the power $k$ any weight appearing in $g_{v}\left(x_{1}, x_{2}, x_{3}, \ldots\right)$ and by multiplying the index of any variable by $k$. The following general principle follows immediately from the equations (23)-(26):

Principle 2.1 Any combinatorial equation (that is, a natural isomorphism, see [2]) between species $F, G, H, \ldots$ gives rise-automatically - to seven corresponding equations between the series

$$
\begin{array}{lll} 
& F(x), G(x), H(x), \ldots, \\
Z_{F}, Z_{G}, Z_{H}, \ldots, & \widetilde{F}(x), \widetilde{G}(x), \widetilde{H}(x), \ldots, & F(x ; q), G(x ; q), H(x ; q), \ldots, \\
\Gamma_{F}, \Gamma_{G}, \Gamma_{H}, \ldots, & \bar{F}(x), \bar{G}(x), \bar{H}(x), \ldots, & F\langle x ; q\rangle, G\langle x ; q\rangle, H\langle x ; q\rangle, \ldots
\end{array}
$$

Here is the main result of this article.

Theorem 2.2 There exists a "universal" virtual weighted species, $\Lambda^{(\alpha)}$, such that for any species of the form $F_{w}=E\left(F_{w}^{c}\right)$, we have

$$
F_{w^{(\alpha)}}=\Lambda^{(\alpha)} \circ F_{w}^{+},
$$

where $F_{w}^{+}=F_{w}-1$ denotes the species of non-empty $F_{w^{-}}$-structures and $F_{w^{(\alpha)}}=E\left(F_{\alpha w}^{c}\right)$ denotes the species of $F_{w}$-structures with the adjunction to the weight of a counter $\alpha$ of connected components. The main series associated to $\Lambda^{(\alpha)}$ are given by the formulas

$$
\begin{gathered}
\Lambda^{(\alpha)}(x)=(1+x)^{\alpha}, \\
Z_{\Lambda^{(\alpha)}}=\prod_{n \geq 1}\left(1+x_{n}\right)^{\lambda_{n}(\alpha)}, \quad \widetilde{\Lambda^{(\alpha)}}(x)=\prod_{n \geq 1}\left(1+x^{n}\right)^{\lambda_{n}(\alpha)}, \quad \Lambda^{(\alpha)}(x ; q)=\prod_{n \geq 1}\left(1+\frac{(1-q)^{n}}{\left(1-q^{n}\right)} x^{n}\right)^{\lambda_{n}(\alpha)}(30) \\
\Gamma_{\Lambda^{(\alpha)}}=\prod_{n \geq 1}\left(1+x_{n}\right)^{\gamma_{n}(\alpha)}, \quad \widetilde{\Lambda^{(\alpha)}}(x)=\prod_{n \geq 1}\left(1+x^{n}\right)^{\gamma_{n}(\alpha)}, \quad \Lambda^{(\alpha)}\langle x ; q\rangle=\prod_{n \geq 1}\left(1+\frac{(1-q)^{n}}{\left(1-q^{n}\right)} x^{n}\right)^{\gamma_{n}(\alpha)},
\end{gathered}
$$

where the exponents $\lambda_{n}(\alpha)$ and $\gamma_{n}(\alpha)$ are polynomials of degree $n$ in $\alpha$ defined by

$$
\begin{aligned}
\lambda_{n}(\alpha) & =\frac{1}{n} \sum_{d \mid n} \mu(n / d) \alpha^{d} \\
\gamma_{n}(\alpha) & =-\lambda_{n}(-\alpha)-\lambda_{n / 2}(-\alpha)-\lambda_{n / 4}(-\alpha)-\cdots-\lambda_{n / 2^{k}}(-\alpha)-\cdots
\end{aligned}
$$

In (31), $\mu$ denotes the classical Möbius function and in (32), the sum ranges over all integers $k \geq 0$ for which $n / 2^{k}$ is an integer. 
Before proving Theorem 2.2, observe that when $\alpha$ is a positive integer, $\lambda_{n}(\alpha)$, given by (31), is equal to the number of primitive circular words (Lyndon words) of length $n$ over an alphabet of $\alpha$ letters (c.f. [29]). It also represents the number of irreducible unitary polynomials of degree $n$ over the finite field $F_{q}$ of cardinality $q$, when $\alpha=q$ (c.f. [11], [31]). The polynomials (31) are known as Lyndon polynomials (see [34]) and also as (primitive) necklace polynomials (see [30]). Finally, note that formula (29) permits the extension of the transformation $F_{w} \mapsto F_{w^{(\alpha)}}$ to all the weighted species $F_{w}$ for which $F_{w}(0)=1$. Indeed, it suffices to substitute $F_{w}^{+}=F_{w}-1$ into $\Lambda^{(\alpha)}$. The species $F_{w}$ can even be virtual. In fact, as long as $F_{w}(0)=1$, a concept of connected components can be defined (see (34) below) and the associated series can be computed (see [2], [20], [22], [25]).

Proof of Theorem 2.2. Consider the species $X_{\alpha}$ of singletons of weight $\alpha$ and $E^{+}=E-1$, of non-empty sets. A. Joyal [20] has shown that the species $E^{+}$possesses an inverse $\left(E^{+}\right)^{<-1>}$ under substitution, given by the formula

$$
\left(E^{+}\right)^{<-1>}=X-\Delta X+\Delta^{2} X-\Delta^{3} X+\cdots,
$$

where $X$ is the species of singletons (of weight 1) and $\Delta$ is the combinatorial difference operator defined by $\Delta G=G \circ E^{+}-G$, for any (virtual) species $G$. Since $F_{w}=E\left(F_{w}^{c}\right)=1+E^{+}\left(F_{w}^{c}\right)$, we get $F_{w}^{+}=F_{w}-1=E^{+}\left(F_{w}^{c}\right)$, whence

$$
F_{w}^{c}=\left(E^{+}\right)^{<-1>} \circ F_{w}^{+}
$$

Let us define $\Lambda^{(\alpha)}$ by the formula

$$
\Lambda^{(\alpha)}:=E \circ X_{\alpha} \circ\left(E^{+}\right)^{<-1>} .
$$

A short computation, based on (33), gives the first few terms of the molecular expansion of $\Lambda^{(\alpha)}$ (see also Section 4):

$$
\Lambda^{(\alpha)}=1+X_{\alpha}+\left(E_{2}\right)_{\alpha^{2}}-\left(E_{2}\right)_{\alpha}+\cdots,
$$

where $\left(E_{2}\right)_{\alpha}$ and $\left(E_{2}\right)_{\alpha^{2}}$ denote the species of sets of cardinality 2 weighted by $\alpha$ and by $\alpha^{2}$, respectively. This shows that $\Lambda^{(\alpha)}$ is a virtual weighted species. We then have successively

$$
F_{w^{(\alpha)}}=E\left(F_{\alpha w}^{c}\right)=E \circ X_{\alpha} \circ F_{w}^{c}=E \circ X_{\alpha} \circ\left(E^{+}\right)^{<-1>} \circ F_{w}^{+}=\Lambda^{(\alpha)} \circ F_{w}^{+}
$$

which gives (29). By taking the generating series of both sides of (35), we get

$$
\Lambda^{(\alpha)}(x)=e^{\alpha \log (1+x)}=(1+x)^{\alpha} .
$$

The explicit formula for the cycle index series $Z_{\Lambda^{(\alpha)}}$ is obtained by substituting the known expressions (see [19], [20], [22])

$$
Z_{E}=\exp \left(\sum_{n \geq 1} x_{n} / n\right), \quad Z_{\left(E^{+}\right)^{<-1>}}=\sum_{n=1}^{\infty} \frac{\mu(n)}{n} \log \left(1+x_{n}\right)
$$

in the formula $Z_{\Lambda^{(\alpha)}}=Z_{E \circ X_{\alpha} \circ\left(E^{+}\right)^{<-1>}}=\left(Z_{E}\right) \circ\left(\alpha x_{1}\right) \circ\left(Z_{\left(E^{+}\right)^{<-1>}}\right)$. The explicit computation of the asymmetry index series $\Gamma_{\Lambda^{(\alpha)}}=\Gamma_{E \circ X_{\alpha} \circ\left(E^{+}\right)^{<-1>}}=\left(\Gamma_{E}\right) \circ\left(\alpha x_{1}\right) \circ\left(\Gamma_{\left(E^{+}\right)<-1>}\right)$ is more delicate. It is known [23] that

$$
\Gamma_{E}=\exp \left(\sum_{n \geq 1}(-1)^{n-1} x_{n} / n\right)
$$


and we must determine $\Theta:=\Gamma_{\left(E^{+}\right)^{<-1>}}$. Since $E \circ\left(E^{+}\right)^{<-1>}=1+E^{+} \circ\left(E^{+}\right)^{<-1>}=1+X$, we have $\Gamma_{E} \circ \Theta=1+x_{1}$. Substituting $\Theta$ in both sides of (40) and taking logarithms, we find

$$
\Theta_{1}-\frac{1}{2} \Theta_{2}+\frac{1}{3} \Theta_{3}-\cdots+\frac{(-1)^{i-1}}{i} \Theta_{i}+\cdots=\log \left(1+x_{1}\right)
$$

where $\Theta_{i}=\Theta\left(x_{i}, x_{2 i}, x_{3 i}, \cdots\right), i \geq 1$. By summation, we obtain the remarkable relation

$$
\Theta_{1}-\Theta_{2}=\Omega, \quad \text { where } \quad \Omega:=Z_{\left(E^{+}\right)^{<-1>}}=\sum_{k=1}^{\infty} \frac{\mu(k)}{k} \log \left(1+x_{k}\right)
$$

since the Möbius function satisfies the property

$$
\frac{1}{n} \sum_{d \mid n} \mu(n / d)(-1)^{d-1}=\left\{\begin{array}{rll}
1, & \text { if } & n=1 \\
-1, & \text { if } & n=2 \\
0, & \text { if } & n \geq 3
\end{array}\right.
$$

Another summation, based on (42), gives

$$
\Theta=\Omega_{1}+\Omega_{2}+\Omega_{4}+\cdots+\Omega_{2^{i}}+\cdots
$$

We then get, by collecting terms properly,

$$
\Gamma_{\Lambda^{(\alpha)}}=\left(\Gamma_{E}\right) \circ\left(\alpha x_{1}\right) \circ\left(\Gamma_{\left(E^{+}\right)<-1>}\right)=\Gamma_{E} \circ\left(\alpha \Omega_{1}+\alpha \Omega_{2}+\alpha \Omega_{4}+\cdots\right)=\prod_{n \geq 1}\left(1+x_{n}\right)^{\gamma_{n}(\alpha)}
$$

where

$$
\gamma_{n}(\alpha)=\sum_{2^{i} \cdot j \cdot k=n} \frac{(-1)^{j-1} \mu(k)}{k j} \alpha^{j}
$$

and equation (32) is satisfied. The other series associated to $\Lambda_{(\alpha)}$ follow directly from the formulas for $Z_{\Lambda_{(\alpha)}}$ and $\Gamma_{\Lambda_{(\alpha)}}$.

The following corollary gives the general explicit form of the series associated to the species $F_{w^{(\alpha)}}$ in terms of the series for $F_{w}$. The proof is straightforward and left to the reader.

Corollary 2.3 Let $F_{w}$ be a weighted virtual species such that $F_{w}(0)=1$ and set $F_{w^{(\alpha)}}=\Lambda^{(\alpha)} \circ F_{w}^{+}$. Then we have

$$
\begin{aligned}
F_{w^{(\alpha)}}(x) & =F_{w}(x)^{\alpha} \\
Z_{F_{w^{(\alpha)}}}\left(x_{1}, x_{2}, x_{3}, \ldots\right) & =\prod_{n \geq 1} Z_{F_{w^{n}}}\left(x_{n}, x_{2 n}, x_{3 n}, \ldots\right)^{\lambda_{n}(\alpha)}, \\
\widetilde{F_{w^{(\alpha)}}}(x) & =\prod_{n \geq 1} \widetilde{F_{w^{n}}}\left(x^{n}\right)^{\lambda_{n}(\alpha)}, \\
F_{w^{(\alpha)}}(x ; q) & =\prod_{n \geq 1} F_{w^{n}}\left(\frac{(1-q)^{n}}{\left(1-q^{n}\right)} x^{n} ; q^{n}\right)^{\lambda_{n}(\alpha)} \\
\Gamma_{F_{w^{(\alpha)}}}\left(x_{1}, x_{2}, x_{3}, \ldots\right) & =\prod_{n \geq 1} \Gamma_{F_{w^{n}}}\left(x_{n}, x_{2 n}, x_{3 n}, \ldots\right)^{\gamma_{n}(\alpha)}, \\
\overline{F_{w^{(\alpha)}}}(x) & =\prod_{n \geq 1} \overline{F_{w^{n}}}\left(x^{n}\right)^{\gamma_{n}(\alpha)}, \\
F_{w^{(\alpha)}}\langle x ; q\rangle & =\prod_{n \geq 1} F_{w^{n}}\left\langle\frac{(1-q)^{n}}{\left(1-q^{n}\right)} x^{n} ; q^{n}\right\rangle^{\gamma_{n}(\alpha)}
\end{aligned}
$$


The special case $\alpha=-1$ is particularly interesting. In the simplest case of the series (47), it corresponds to the computation of the excess weight of the $F_{w}$-structures having an even number of connected components over those having an odd number:

$$
F_{w^{(-1)}}(x)=\sum_{n \geq 0}\left(f_{n}^{+}-f_{n}^{-}\right) \frac{x^{n}}{n !}
$$

where

$$
\begin{aligned}
& f_{n}^{+}=\text {total weight of } F_{w} \text {-structures on }[n] \text { with an even number of connected components, } \\
& f_{n}^{-}=\text {total weight of } F_{w^{-}} \text {-structures on }[n] \text { with an odd number of connected components. }
\end{aligned}
$$

The behavior of the seven main series with respect to the transformation $F_{w} \mapsto F_{w^{(-1)}}$ is described in the following corollary.

Corollary 2.4 Let $F_{w}$ be a weighted virtual species such that $F_{w}(0)=1$ and set $F_{w^{(-1)}}=\Lambda^{(-1)} \circ F_{w}^{+}$. Then we have

$$
\begin{aligned}
F_{w^{(-1)}}(x) & =1 / F_{w}(x), \\
Z_{F_{w}(-1)}\left(x_{1}, x_{2}, x_{3}, \ldots\right) & =\frac{Z_{F_{w^{2}}}\left(x_{2}, x_{4}, x_{6}, \ldots\right)}{Z_{F_{w}}\left(x_{1}, x_{2}, x_{3}, \ldots\right)}, \\
\widetilde{F_{w^{(-1)}}}(x) & =\frac{\widetilde{F_{w^{2}}}\left(x^{2}\right)}{\widetilde{F_{w}}(x)}, \\
F_{w^{(-1)}}(x ; q) & =\frac{F_{w^{2}}\left(\frac{(1-q)}{(1+q)} x^{2} ; q^{2}\right)}{F_{w}(x ; q)}, \\
\Gamma_{F_{w}(-1)}\left(x_{1}, x_{2}, x_{3}, \ldots\right) & =1 / \prod_{k \geq 0} \Gamma_{F_{w^{2}}}\left(x_{2^{k}}, x_{2 \cdot 2^{k}}, x_{3 \cdot 2^{k}}, \ldots\right), \\
\overline{F_{w^{(-1)}}}(x) & =1 / \prod_{k \geq 0} \overline{F_{w^{2}}}\left(x^{2^{k}}\right), \\
F_{w^{(-1)}}\langle x ; q\rangle & =1 / \prod_{k \geq 0} F_{w^{2}}\left\langle\frac{(1-q)^{2^{k}}}{\left(1-q^{2^{k}}\right)} x^{2^{k}} ; q^{2^{k}}\right\rangle .
\end{aligned}
$$

Proof. It suffices to use the relations

$$
\lambda_{n}(-1)=\left\{\begin{aligned}
-1, & \text { if } \quad n=1 \\
1, & \text { if } n=2 \\
0, & \text { if } \quad n \geq 3
\end{aligned} \quad \text { and } \quad \gamma_{n}(-1)=\left\{\begin{aligned}
-1, & \text { if } n=2^{k}, k \geq 0 \\
0, & \text { otherwise }
\end{aligned}\right.\right.
$$

Observe that the transformations described by (57)-(63) are involutive since $w^{(-1)(-1)}=w$. It is easily verified that the series associated to the universal virtual species $\Lambda^{(-1)}$ are given by

$$
\Lambda^{(-1)}(x)=(1+x)^{-1},
$$




$$
\begin{array}{rlrl}
Z_{\Lambda^{(-1)}}=\frac{1+x_{2}}{1+x_{1}}, & \widetilde{\Lambda^{(-1)}}(x)=\frac{1+x^{2}}{1+x}, & \Lambda^{(-1)}(x ; q) & =\frac{1+\frac{1-q}{1+q} x^{2}}{1+x}, \\
\Gamma_{\Lambda^{(-1)}}=\prod_{k \geq 0}\left(1+x_{2^{k}}\right)^{-1}, & \widetilde{\Lambda^{(-1)}}(x)=1-x, & \Lambda^{(-1)}\langle x ; q\rangle=\prod_{k \geq 0}\left(1+\frac{(1-q)^{2^{k}}}{1-q^{2^{k}}} x^{2^{k}}\right)^{-1} .
\end{array}
$$

\section{Examples arising from particular choices of $F_{w}$}

The corollaries 2.3 and 2.4 give rise to a multitude of identities and of interesting expressions, by choosing particular species $F_{w}$. For example, in the case of the species $F_{w}=E$, of sets (trivially weighted by $w=1)$, we have $Z_{E}=\exp \left(\sum_{n \geq 1} x_{n} / n\right)$ and $\Gamma_{E}=\exp \left(\sum_{n \geq 1}(-1)^{n-1} x_{n} / n\right)$. The formulas (47)-(53) of Corollary 2.3 produce respectively the identities

$$
\begin{aligned}
e^{\alpha x} & =\left(e^{x}\right)^{\alpha}, \\
\exp \left(\sum_{k \geq 1} \alpha^{k} x_{k} / k\right) & =\prod_{n \geq 1} \exp \left(\lambda_{n}(\alpha) \sum_{k \geq 1} x_{k n} / k\right), \\
(1-\alpha x)^{-1} & =\prod_{n \geq 1}\left(1-x^{n}\right)^{-\lambda_{n}(\alpha)}, \\
E(\alpha x ; q) & =E(x ; q)^{\alpha} E\left(\frac{(1-q)^{2}}{\left(1-q^{2}\right)} x^{2} ; q^{2}\right)^{\lambda_{2}(\alpha)} E\left(\frac{(1-q)^{3}}{\left(1-q^{3}\right)} x^{3} ; q^{3}\right)^{\lambda_{3}(\alpha)} \ldots, \\
\exp \left(\sum_{k \geq 1}(-1)^{k-1} \alpha^{k} x_{k} / k\right) & =\prod_{n \geq 1} \exp \left(\lambda_{n}(\alpha) \sum_{k \geq 1}(-1)^{k-1} x_{k n} / k\right), \\
1+\alpha x & =\prod_{n \geq 1}\left(1+x^{n}\right)^{\gamma_{n}(\alpha)}, \\
E\langle\alpha x ; q\rangle & =E\langle x ; q\rangle^{\alpha} E\left\langle\frac{(1-q)^{2}}{\left(1-q^{2}\right)} x^{2} ; q^{2}\right\rangle^{\gamma_{2}(\alpha)} E\left\langle\frac{(1-q)^{3}}{\left(1-q^{3}\right)} x^{3} ; q^{3}\right\rangle^{\gamma_{3}(\alpha)} \ldots .
\end{aligned}
$$

The reader will recognize, in (68), the classical cyclotomic identity of Gauss (see [10], [30]). The identity (71) constitutes a kind of dual of (68) and could be called the cocyclotomic identity. The identities (67) (due to F. Bergeron, see [1], p. 300) and (70) are extensions of (68) and (71) which also yield (69) and (72) by specialization. Expressed in terms of the $q$-exponentials, the latter can be seen as $q$-analogues of the cyclotomic and cocyclotomic identities.

In the case of the species $F_{w}=S$, of permutations, we have $Z_{S}=\prod_{n \geq 1}\left(1-x_{n}\right)^{-1}$ and $\Gamma_{S}=$ $\left(1-x_{2}\right) /\left(1-x_{1}\right)$. Let us then denote by $S_{(\alpha)}$, the transformed species of permutations, weighted by the cycle counter $\alpha$. Applications of Corollary 2.3 give for example, after a proper term collection,

$$
Z_{S_{(\alpha)}}=\prod_{n \geq 1}\left(1-x_{n}\right)^{-\nu_{n}(\alpha)}
$$

where $\nu_{n}(\alpha)$ denotes the polynomial defined by

$$
\nu_{n}(\alpha)=\frac{1}{n} \sum_{d \mid n} \phi(n / d) \alpha^{d},
$$

where $\phi(n)$ is the Euler totient function. When $\alpha$ is a positive integer, $\nu_{n}(\alpha)$ is the number of circular words of length $n$ over an alphabet with $\alpha$ letters or, equivalently, the number of (not necessarily primitive) necklaces of length $n$ with $\alpha$ colors. Formula (73), due to H. Décoste, is the basis for the

computation of the cycle index series of combinatorial models of the classical orthogonal polynomials 
of Laguerre, Charlier, Meixner, Meixner-Pollaczek, and Jacobi, (see [7], [8], and also [6], [34] and [35]) and their canonical $q$-analogues. We also obtain

$$
\Gamma_{S_{(\alpha)}}=\prod_{n \text { odd }}\left(1-x_{n}\right)^{-\gamma_{n}(\alpha)} \prod_{n \text { even }}\left(1-x_{n}\right)^{\lambda_{n}(-\alpha)} .
$$

Taking the particular value $\alpha=-1$, Corollary 2.4 gives the following formulas:

$$
\begin{aligned}
S_{(-1)}(x) & =1-x, \\
Z_{S_{(-1)}}\left(x_{1}, x_{2}, x_{3}, \ldots\right) & =\left(1-x_{1}\right)\left(1-x_{3}\right)\left(1-x_{5}\right) \cdots, \\
\widetilde{S_{(-1)}}(x) & =(1-x)\left(1-x^{3}\right)\left(1-x^{5}\right) \cdots, \\
S_{(-1)}(x ; q) & =(1-x)\left(1-\frac{(1-q)^{3}}{\left(1-q^{3}\right)} x^{3}\right)\left(1-\frac{(1-q)^{5}}{\left(1-q^{5}\right)} x^{5}\right) \cdots, \\
\Gamma_{S_{(-1)}}\left(x_{1}, x_{2}, x_{3}, \ldots\right) & =1-x_{1}, \\
\overline{S_{(-1)}}(x) & =1-x, \\
S_{(-1)}\langle x ; q\rangle & =1-x .
\end{aligned}
$$

The reader is invited to give direct combinatorial proofs of these formulas.

In the case of the species $F_{w}=\mathrm{Gr}_{w}$ of graphs, weighted by an edge counter $y$, already seen in the introduction, the series $\mathrm{Gr}_{w^{(\alpha)}}(x), Z_{\mathrm{Gr}_{w}^{(\alpha)}}, \widetilde{\mathrm{Gr}_{w^{(\alpha)}}}(x), \mathrm{Gr}_{w^{(\alpha)}}(x ; q)$, of the associated species $\mathrm{Gr}_{w^{(\alpha)}}$ can be computed with the help of (47)-(50), by using the known formula (see [2], [18])

$$
Z_{\mathrm{Gr}}\left(x_{1}, x_{2}, x_{3}, \ldots\right)=\sum_{n=0}^{\infty} \frac{1}{n !} \sum_{\sigma \in S_{n}}(1+y)^{c_{1}}\left(1+y^{2}\right)^{c_{2}}\left(1+y^{3}\right)^{c_{3}} \cdots x_{1}^{\sigma_{1}} x_{2}^{\sigma_{2}} x_{3}^{\sigma_{3}} \cdots
$$

where

$$
c_{k}=\frac{1}{2} \sum_{[i, j]=k}(i, j) \sigma_{i} \sigma_{j}+\sigma_{2 k}-\sigma_{k}+\frac{1}{2}(k \bmod 2) \sigma_{k},
$$

and where $[i, j]$ denotes the smallest common multiple of the integers $i$ and $j$. However, the explicit computation of the other series $\Gamma_{\mathrm{Gr}_{w}(\alpha)}, \overline{\mathrm{Gr}_{w^{(\alpha)}}}(x), \mathrm{Gr}_{w^{(\alpha)}}\langle x ; q\rangle$ is an open problem since no closed formulas for the series $\Gamma_{\mathrm{Gr}_{w}}, \overline{\mathrm{Gr}_{w}}(x), \mathrm{Gr}_{w}\langle x ; q\rangle$ are known up to now.

Other applications are possible, in addition to the various combinatorial models of orthogonal polynomials already mentioned. An example is given by the species $F_{w}=E(A)=A / X$, of forests of rooted trees (where $A$ is the species of rooted trees). The transformation $F_{w} \mapsto F_{w^{(\alpha)}}$ is then equivalent to the introduction of a counter for trees in the forests. Taking the coefficients of the seven associated series yields families of polynomials in $\alpha: a_{n}(\alpha), a_{\sigma}(\alpha), \tilde{a}_{n}(\alpha), a_{n}(\alpha ; q), a_{\sigma}^{*}(\alpha), \bar{a}_{n}(\alpha)$ and $a_{n}\langle\alpha ; q\rangle$. The first is the family of classical Abel polynomials $a_{n}(\alpha)=\alpha(\alpha+n)^{n-1}$ while the others constitute generalized versions of it. Note that these generalized Abel polynomials are distinct from those introduced in [26], since the species $F_{w^{(\alpha)}}$ is different from the simple exponentiation $\left(F_{w}\right)^{\alpha}$, even when $\alpha$ is an integer.

\section{Some properties of the species $\Lambda^{(\alpha)}$}

Recall that the universal virtual weighted species $\Lambda^{(\alpha)}$ is defined by $\Lambda^{(\alpha)}=E \circ X_{\alpha} \circ\left(E^{+}\right)^{<-1>}$ and that we have $F_{w^{(\alpha)}}=\Lambda^{(\alpha)} \circ F_{w}^{+}$, for any weighted species $F_{w}$ such that $F_{w}(0)=1$. Since the exponential generating series of $\Lambda^{(\alpha)}$ given by $\Lambda^{(\alpha)}(x)=(1+x)^{\alpha}$, it is natural to ask which properties of the function 
$(1+x)^{\alpha}$ carry over to the species $\Lambda^{(\alpha)}$. For example, the algebraic identity $\left((1+x)^{\beta}\right)^{\alpha}=(1+x)^{\alpha \beta}$ can be lifted to the combinatorial equality

$$
\Lambda^{(\alpha)} \circ\left(\Lambda^{(\beta)}\right)^{+}=\Lambda^{(\alpha \beta)}
$$

where $\left(\Lambda^{(\beta)}\right)^{+}=\Lambda^{(\beta)}-1=E^{+} \circ X_{\beta} \circ\left(E^{+}\right)^{<-1>}$ is the conjugate (for composition) of the species of singletons of weight $\beta$ by that of non-empty sets. Indeed, since $X_{\alpha} \circ X_{\beta}=X_{\alpha \beta}$, we have immediately

$$
E \circ X_{\alpha} \circ\left(E^{+}\right)^{<-1>} \circ E^{+} \circ X_{\beta} \circ\left(E^{+}\right)^{<-1>}=E \circ X_{\alpha \beta} \circ\left(E^{+}\right)^{<-1>} .
$$

By taking the cycle and asymmetry index series of both sides of (85) the following identities are easily obtained:

$$
\lambda_{n}(\alpha \beta)=\sum_{i \cdot j=n} \lambda_{i}\left(\alpha^{j}\right) \lambda_{j}(\beta), \quad \gamma_{n}(\alpha \beta)=\sum_{i \cdot j=n} \gamma_{i}\left(\alpha^{j}\right) \gamma_{j}(\beta), \quad n \geq 0
$$

The identity on the left hand side of (87) is a variant of the well known relation (see [30])

$$
\lambda_{n}(\alpha \beta)=\sum_{[i, j]=n}(i, j) \lambda_{i}(\alpha) \lambda_{j}(\beta)
$$

Of course, the algebraic identity $\Lambda^{(\alpha)}(x) \cdot \Lambda^{(\beta)}(x)=(1+x)^{\alpha}(1+x)^{\beta}=(1+x)^{\alpha+\beta}=\Lambda^{(\alpha+\beta)}(x)$, between generating series, is satisfied. However, we have, at the species level,

$$
\Lambda^{(\alpha)} \cdot \Lambda^{(\beta)} \neq \Lambda^{(\alpha+\beta)}
$$

This behavior follows from the fact that $X_{\alpha}+X_{\beta} \neq X_{\alpha+\beta}$. It is also reflected by the fact that although $\lambda_{1}(\alpha+\beta)=\alpha+\beta=\lambda_{1}(\alpha)+\lambda_{1}(\beta)$, we have

$$
\lambda_{n}(\alpha+\beta) \neq \lambda_{n}(\alpha)+\lambda_{n}(\beta), \quad \text { if } n \geq 2 .
$$

The difference operator $\Delta$, defined by $\Delta G=G \circ E^{+}-G$, permits the expression of $\Lambda^{(\alpha)}$ in the form

$$
\Lambda^{(\alpha)}=E\left(X_{\alpha}\right)-\Delta E\left(X_{\alpha}\right)+\Delta^{2} E\left(X_{\alpha}\right)-\Delta^{3} E\left(X_{\alpha}\right)+\cdots
$$

By using the computer algebra software Maple [4], Y. Chiricota (personal communication, see also [5]) has computed the molecular decomposition of $\Lambda^{(\alpha)}$, i.e., its expression as a $\mathbb{Z}$-linear combination of molecular (i.e., indecomposable under addition) species, up to degree 7 . Here are the first terms, up to degree 5:

$$
\begin{aligned}
\Lambda^{(\alpha)}= & E \circ X_{\alpha} \circ\left(E^{+}\right)^{<-1>} \\
=1 & +X_{\alpha}-\left(E_{2}\right)_{\alpha}+\left(E_{2}\right)_{\alpha^{2}}-\left(E_{3}\right)_{\alpha}+\left(X E_{2}\right)_{\alpha}-\left(X E_{2}\right)_{\alpha^{2}}+\left(E_{3}\right)_{\alpha^{3}} \\
& +\left(E_{2} \circ E_{2}\right)_{\alpha}-\left(E_{4}\right)_{\alpha}+\left(X E_{3}\right)_{\alpha}-\left(X^{2} E_{2}\right)_{\alpha} \\
& +\left(E_{2}^{2}\right)_{\alpha^{2}}-\left(X E_{3}\right)_{\alpha^{2}}+\left(X^{2} E_{2}\right)_{\alpha^{2}}-\left(E_{2} \circ E_{2}\right)_{\alpha^{2}}-\left(E_{2}^{2}\right)_{\alpha^{3}}+\left(E_{4}\right)_{\alpha^{4}} \\
& +\left(E_{2} E_{3}\right)_{\alpha}+\left(X E_{4}\right)_{\alpha}+\left(X^{3} E_{2}\right)_{\alpha}-\left(X^{2} E_{3}\right)_{\alpha}-\left(X E_{2}^{2}\right)_{\alpha}-\left(E_{5}\right)_{\alpha} \\
& +\left(E_{2} E_{3}\right)_{\alpha^{2}}+\left(X^{2} E_{3}\right)_{\alpha^{2}}-\left(X E_{4}\right)_{\alpha^{2}}-\left(X^{3} E_{2}\right)_{\alpha^{2}}+\left(X E_{2} \circ E_{2}\right)_{\alpha^{2}}-\left(X E_{2}^{2}\right)_{\alpha^{2}} \\
& +2\left(X E_{2}^{2}\right)_{\alpha^{3}}-\left(E_{2} E_{3}\right)_{\alpha^{3}}-\left(X E_{2} \circ E_{2}\right)_{\alpha^{3}}-\left(E_{2} E_{3}\right)_{\alpha^{4}}+\left(E_{5}\right)_{\alpha^{5}} \\
& +\cdots,
\end{aligned}
$$


where $E_{k}$ denotes the species of sets of cardinality $k$. The molecular decomposition (92) can be seen as a combinatorial lifting of the binomial formula

$$
\begin{aligned}
\Lambda^{(\alpha)}(x) & =(1+x)^{\alpha} \\
& =1+\alpha x+\alpha(\alpha-1) \frac{x^{2}}{2 !}+\alpha(\alpha-1)(\alpha-2) \frac{x^{3}}{3 !}+\alpha(\alpha-1)(\alpha-2)(\alpha-3) \frac{x^{4}}{4 !}+\cdots .
\end{aligned}
$$

The formulas (91) and (92) show that $\Lambda^{(\alpha)}$ is a species of set-like type: all its molecular components are obtained by products and compositions of species of the form $E_{k}$. For all $n \geq 0$, set

$$
\begin{aligned}
m_{n} & =\text { number of set-like molecular species of degree } n \\
a_{n} & =\text { number of set-like atomic species of degree } n
\end{aligned}
$$

A species is atomic if it is molecular and indecomposable under the product. It can be shown that the sequences $\left(m_{n}\right)_{n \geq 0}$ and $\left(a_{n}\right)_{n \geq 0}$ satisfy the double recursive scheme

$$
\begin{array}{ll}
m_{0}=1, & m_{1}=1, \\
a_{0}=0, & a_{1}=1,
\end{array} \quad \begin{cases}m_{n}=\frac{1}{n} \sum_{k=1}^{n}\left(\sum_{d \mid k} d a_{d}\right) m_{n-k} & \\
a_{n}=\sum_{\substack{k \mid n \\
k<n}} m_{k} & n \geq 2,\end{cases}
$$

which is equivalent to the following relations:

$$
\sum_{n \geq 0} m_{n} x^{n}=\prod_{k \geq 1} \frac{1}{\left(1-x^{k}\right)^{a_{k}}}, \quad \sum_{n \geq 1} \frac{a_{n}}{n^{s}}=1+(\zeta(s)-1) \cdot \sum_{n \geq 1} \frac{m_{n}}{n^{s}}
$$

where $\zeta(s)$ denotes the Riemann zeta function. In the case of all atomic and molecular species, the first of these relations has an analogue (see [27], [28]) but not the second one. Here are the values of $m_{n}$ and $a_{n}$, for $0 \leq n \leq 40$ :

$$
\begin{aligned}
m_{n}= & 1,1,2,3,7,9,20,26,54,74,137,184,356,473,841,1154,2034,2742,4740,6405,10874 \\
& 14794,24515,33246,54955,74380,120501,163828,263144,356621,567330,768854,1212354, \\
& 1644335,2567636,3478873,5403223,7314662,11265825,15258443,23363143 \\
a_{n}= & \quad 0,1,1,1,3,1,6,1,10,4,12,1,33,1,29,13,64,1,100,1,156,30,187,1,443,10,476,78 \\
& 877,1,1326,1,2098,188,2745,36,5203,1,6408,477,11084 .
\end{aligned}
$$

These sequences do not appear in the 1973 edition of Sloane's book [32] but they do in the new edition [33]. The analytical properties of the functions (97) should prove useful for the asymptotic analysis of the numbers $m_{n}$, for $n \rightarrow \infty$.

\section{References}

[1] F. Bergeron, Une combinatoire du pléthysme. Journal of Combinatorial Theory, Series A, 46 (1987), 291-305.

[2] F. Bergeron, G. Labelle et P. Leroux, Théorie des espèces et combinatoire des structures arborescentes. Publications du LACIM, Vol. 19, Université du Québec à Montréal, 1994. 
[3] P. Cartier et D. Foata, Problèmes combinatoires de commutation et de réarrangements. Lecture Notes in Mathematics, 85, Springer-Verlag, 1969.

[4] B. W. Char, K. O. Geddes, G. H. Gonnet, B. L. Leong, M. B. Monagan and S. M. WATT, MAPLE V Language Reference Manual. Springer-Verlag, 1991.

[5] Y. Chiricota, Structures combinatoires et calcul symbolique. Publications du LACIM, Vol. 12, Université du Québec à Montréal, 1993.

[6] I. Constantineau, Auto-similarité dans la combinatoire des polynômes orthogonaux. Theoretical Computer Science, Elsevier, 117 (1993), 153-167.

[7] H. DÉcoste, Séries indicatrices d'espèces pondérées et q-analogues. Publications du LACIM, Vol. 2, Université du Québec à Montréal, 1990 (Ph.D. Thesis, Un. de Montréal, 1989).

[8] H. DÉcoste, Séries indicatrices et q-séries. Theoretical Computer Science, Elsevier, 117 (1993), 169-186.

[9] H. Décoste et G. Labelle, Le q-dénombrement générique d'une espèce de structures. Discrete Mathematics, à paraître.

[10] A. Dress And C. Siebeneicher, The Burnside ring of the infinite cyclic group and its relations to the necklace algebra, lambda-rings, and the universal ring of Witt vectors, Adv. in Mathematics, 78 (1989) 1-41.

[11] A. Dress And C. Siebeneicher On the number of solutions of certain linear Diophantine equations. Hokkaido Mathematical Journal, 19 (1990), 385-401.

[12] D. Foata, La série exponentielle dans les problèmes d'énumération. Presses de l'Université de Montréal, 1974.

[13] D. FoATA, A combinatorial proof of the Mehler formula. Journal of Combinatorial Theory, Series A, 24 (1978), 367-376.

[14] D. Foata et J. Labelle, Modèles combinatoires pour les polynômes de Meixner. European Journal of Combinatorics, 4 (1983), 305-311.

[15] D. Foata et P. Leroux, Polynômes de Jacobi, interprétation combinatoire et fonction génératrice. Proceedings of the American Mathematical Society, 87 (1983), 47-53.

[16] D. Foata et M. P. Schutzenberger, Théorie géométrique des polynômes eulériens. Lecture Notes in Mathematics, Vol. 138, Springer-Verlag, 1970.

[17] D. Foata and V. Strehl, Combinatorics of Laguerre polynomials. In: D. M. Jackson and S. A. Vanstone, eds., Enumeration and Design, Academic Press, 1984, 123-140.

[18] F. Harary and E. Palmer, Graphical enumeration. Academic Press, 1973.

[19] A. Joyal, Une théorie combinatoire des séries formelles. Advances in Mathematics, 42 (1981), $1-82$.

[20] A. Joyal, Foncteurs analytiques et espèces de structures. In: G. Labelle and P. Leroux, eds, Combinatoire énumérative, Proceedings, Montréal, Québec, 1985. Lecture Notes in Mathematics, Vol. 1234, Springer-Verlag, 1986, 126-159. 
[21] G. LABELle, Une nouvelle démonstration combinatoire des formules d'inversion de Lagrange. Advances in Mathematics, 42 (1981), 217-247.

[22] G. Labelle, Some new computational methods in the theory of species, In: G. Labelle and P. Leroux, eds, Combinatoire énumérative, Proceedings, Montréal, Québec 1985. Lecture Notes in Mathematics, Vol. 1234, Springer-Verlag, 1986, 192-209.

[23] G. Labelle, On asymmetric structures. Discrete Mathematics, 99 (1992), 141-164.

[24] G. Labelle, Counting asymmetric enriched trees. Journal of Symbolic Computation, 14 (1992), $211-242$.

[25] G. LABELle, Sur la symétrie et l'asymétrie des structures combinatoires. Theoretical Computer Science, Elsevier, 117 (1993), 3-22.

[26] G. LABELLE, Enriched q-Abel identities arising from symmetric functions. Rapport de Recherche, Département de Mathématiques, UQAM , no 244, mars 1995.

[27] J. LABELle, Quelques espèces sur les ensembles de petites cardinalités. Annales des sciences mathématiques du Québec, 9 (1985), 31-58.

[28] J. Labelle And Y.-N. YeH, The Relation between Burnside Rings and Combinatorial Species, Jour. Combin. Th., A, 50 (1989), 269-284.

[29] M. Lothaire, Combinatorics on Words. Encyclopedia of Mathematics, Vol. 17, Addison-Wesley 1983, Cambridge University Press 1984.

[30] N. Metropolis And G.-C. Rota, Witt Vectors and the Algebra of Necklaces. Advances in Mathematics, 50 (1983), 95-125.

[31] C. Reutenauer, Mots circulaires et polynômes irréductibles. Annales des sciences mathématiques du Québec, 12 (1988), 275-285.

[32] N. J. A. Slonne, A Handbook of Integer Sequences. Academic Press, 1973.

[33] N. J. A. Sloane And S. Plouffe, The Encyclopedia of Integer Sequences. Academic Press, 1995.

[34] V. Strehl, Cycle counting for isomorphism types of endofunctions. Bayreuther Mathematische Schriften, 40 (1992), 153-167.

[35] V. Strehl, Zykel-Enumeration bei lokal-strukturierten Funktionen. Habilitationsschrift, Institut für Mathematische Maschinen und Datenverarbeitung, Universität Erlangen-Nürnberg, 1990

[36] Y.-N. YEH, The calculus of virtual species and $\mathbb{K}$-species. In: G. Labelle and P. Leroux, eds, Combinatoire énumérative, Proceedings, Montréal, Québec, 1985. Lecture Notes in Mathematics, Vol. 1234, Springer-Verlag, Berlin, 1986, 351-369. 